\title{
El turismo desde un enfoque de sociología constructivista
}

DOI: 10.22403/UQROOMX/TYP06/06

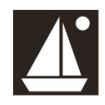

RESUMEN

Alfonso González Damián* Universidad de Quintana Roo

Se presenta una propuesta de aproximación teórica al turismo, desde la sociología constructivista, específicamente desde la construcción de la realidad social de Peter L. Berger y Thomas Luckmann. La finalidad es aportar ideas que favorezcan el debate académico y el diálogo frente a otros acercamientos teóricos, en particular frente al enfoque sistémico, que encabeza las preferencias entre los estudiosos del turismo.

\footnotetext{
$\begin{array}{r}\text { Palabras } \\ \text { CLAVe }\end{array} \mid$ Turismo, sociología constructivista, teoría.
}

*Correo electrónico: gonzalez@uqroo.mx 


\section{Introducción}

La teoría constructivista en la sociología

Como muchos otros fenómenos en la sociedad moderna, el turismo ha sido estudiado por la sociología. Sin embargo, puesto que existen diversas sociologías, y dado que no hay una teoría única de la sociedad que deje satisfechos a todos los sociólogos, es justo decir también que hay variados enfoques que pueden aportar pistas o ángulos para ampliar el conocimiento sobre el turismo.

Hoy día, el término constructivismo es prácticamente omnipresente, y en verdad tiene muy distintos significados según el ámbito en el que se maneje. Michael Matthews dice al respecto que, mientras hay "innumerables artículos constructivistas, es raro encontrar uno con una epistemología totalmente definida, una teoría de aprendizaje, teoría educacional, o posiciones éticas o políticas" (Matthews, 1992).

Para encontrar un orden o un hilo conductor en la vasta producción sobre constructivismo se puede partir de la diferenciación que establece Chadwick (2005), quien afirma que en realidad hay dos constructivismos. El primero, con frecuencia denominado constructivismo social o deconstruccionismo, o incluso posmodernismo, trata sobre los conocimientos desarrollados durante el curso de la historia de la humanidad, que son construcciones humanas organizadas en campos tales como política, ideologías, valores, ejercicio del poder y preservación del estatus, creencias religiosas e interés económico personal. Esta tesis niega que las disciplinas sean el reflejo objetivo de un "mundo externo". El segundo constructivismo, llamado psicológico, consiste en una manera de entender cómo aprenden los individuos, sugiriendo que construyen activamente su propio conjunto de significados o interpretaciones, y que el conocimiento no es una mera copia del mundo externo ni tampoco se adquiere por la absorción pasiva o por la simple transferencia de una persona (maestro) a otra (alumno). Esta postura establece que el conocimiento se construye, no se adquiere.

La propuesta de Berger y Luckmann se inserta en el constructivismo social, concretamente en la sociología del conocimiento, y ha sido, como toda teoría sociológica, objeto de críticas y análisis, aunque sus posibilidades como marco para el estudio del turismo todavía no han sido exploradas con amplitud. 


\section{Antecedentes y metodología}

La construcción social de la realidad en Berger y Luckmann

En su ya clásico tratado sobre sociología del conocimiento, Peter L. Berger y Thomas Luckmann (1968), a pesar de advertir desde el prefacio de su obra que no pretenden promover ningún debate, terminan haciéndolo al sintetizar ideas y planteamientos que provienen de diversas fuentes, pero en particular rinden tributo a las ideas de Alfred Schütz, sobre las cuales elaboran gran parte de sus proposiciones. Los aportes de Berger y Luckmann, que durante mucho tiempo tuvieron un carácter local centrado sobre todo en sus universidades y entre algunos sociólogos interesados en el tema del conocimiento en los Estados Unidos, se han encontrado en años recientes con nuevos lectores y con un auge que seguramente ni los propios autores esperaron cuando escribieron el libro.

Para Berger y Luckmann, la realidad logra su establecimiento en la sociedad y en los individuos, como una consecuencia de un proceso dialéctico entre un elemento "objetivo", que incluye las relaciones sociales, los hábitos tipificados y las estructuras sociales, y un elemento "subjetivo", que integra a las interpretaciones simbólicas, la internalización de roles y la formación de identidades individuales. Consideran que lo que interesa es explicar el modo en que la realidad es construida socialmente y analizar los procesos por los cuales esto se produce. Para ello proponen los elementos que configuran la tríada "realidad interpretada/significado subjetivo/mundo coherente" y que son: la conciencia, que define la intención y la búsqueda de objetos; el mundo intersubjetivo, que se comparte con los demás; la temporalidad, como carácter básico de la conciencia (orden temporal); la interacción social, que crea esquemas tipificadores; $y$ el lenguaje, como elemento clave objetivo (externo al individuo) que facilita la estructuración del conocimiento en términos de relevancia.

Con el fin de construir un planteamiento teórico del turismo desde la sociología constructivista de Berger y Luckmann fue necesario realizar un ejercicio de análisis deductivo, en el que se tomaron las categorías y los conceptos centrales de la teoría sociológica, visualizando sus especificidades para el caso del fenómeno turístico. En este sentido, además de las fuentes y los críticos de la teoría sociológica en cuestión, se requirió revisar los antecedentes y fundamentos teóricos del turismo en las ciencias sociales y en la sociología. 
A continuación se presentan los resultados de este examen para que puedan ser debatidos $y$, en su caso, sirvan como referente para el trabajo académico científico del turismo.

\section{Resultados y discusión}

La interacción, el tiempo y el espacio en el turismo desde una perspectiva de sociología constructivista

El turismo es una actividad que se realiza en sociedad, por lo tanto, existe en la sociedad y, como tal, se elabora mediante la interacción entre los diversos actores que la conforman. Aunque el turismo abarca múltiples aspectos de la realidad, incluidos aquellos psicológicos o internos del individuo y que sólo ocurren en él, hasta los que sólo suceden de manera externa al hombre -como condiciones físicas, químicas, etc. -, aquí sólo se abordará la parte del turismo que sucede en la sociedad.

La parte social del turismo es la que aquí se concibe como una realidad construida.Ahora bien, en cuanto realidad tiene "elementos objetivos", y en cuanto construcción implica que lo "objetivo" se alcanza mediante lo "subjetivo".

La parte social del turismo se construye a partir de interacciones entre personas, de lo cual pueden desprenderse algunas afirmaciones: un único individuo no forma una sociedad. Al parecer, tal declaración no supone demasiadas dudas; para efectos prácticos es poco relevante, pues todo individuo, para subsistir, necesita de otros semejantes, por lo que desde su nacimiento, incluso antes de él, ya está integrado a una sociedad. Sin embargo, la siguiente afirmación puede hacer ver el sentido de la primera: no basta con que existan dos individuos para conformar una sociedad; es requisito que cuenten con los recursos y conocimientos suficientes para establecer una interacción, cuya repetición va construyendo la sociedad. Así, un solo individuo no conforma el turismo, ni tampoco dos personas o dos grupos, es indispensable que dichas personas o grupos cuenten con los recursos y los conocimientos necesarios para establecer interacciones de tipo turístico y que éstas sean repetidas para que, con el tiempo, se erija la realidad social del turismo en determinado sitio o espacio de destino turístico.

El espacio en el que se efectúa la interacción turística es el denominado sitio de destino turístico, haciendo alusión a la región visitada durante un viaje. 
Un destino turístico es, en general, cualquier lugar del mundo; sobre todo hoy, con la expansión de las comunicaciones, vías y caminos, prácticamente cualquier sitio es susceptible de ser visitado por turistas. En términos geoespaciales, un destino turístico no se constriñe a las demarcaciones políticas fijadas por las comunidades, poblaciones, regiones, entidades, países o provincias, sino que obedece a una lógica espacial turística que lo significa como un solo destino. Cualquier sitio que en la actualidad cuente con vías de comunicación es un posible destino para los viajeros turísticos. En este sentido, los espacios del turismo son aquellos en los que se manifiesta la interacción turística a partir de las prácticas de anfitriones como anfitriones y de los turistas como turistas.

Los anfitriones, quienes residen en una localidad visitada por los turistas, perciben como territorios turísticos en su propia comunidad a aquellos espacios en los que se reúnen los turistas: las playas, los parques naturales y recreativos, las plazas públicas, los centros comerciales, los hoteles, los restaurantes de cierta calidad "turística", los aeropuertos, las terminales de autobuses. No todos los espacios son compartidos por el anfitrión con el turismo; existen amplias zonas de las ciudades o regiones que no son consideradas territorio del turista. Estas percepciones de la ciudad como propia -"nuestro" espacio- se van formando en la vida cotidiana, a partir de la experiencia diaria y de los significados que se atribuyen a los propios espacios como espacios tipificados.

Por su parte, para los turistas, el espacio turístico es percibido como aquel por el que libremente pueden transitar y que, si bien consideran distante, les resulta "apropiable" cuando menos durante períodos temporales limitados. El espacio turístico incluye aquellos sitios en los que el turista desarrolla alguna actividades propias de su viaje: alojamiento, alimentación, descanso, recreación. Se le presenta como un paisaje ajeno, pero que puede disfrutar todo el tiempo que dure su estancia, $y$ que se extiende al territorio que recorrió para llegar al lugar de destino, es decir, los caminos y vías terrestres, aéreas y acuáticas que debió transitar para ello. Este conjunto de espacios se transforma en imágenes y momentos que serán evocados como parte de la vivencia del turista en el sitio visitado.

Hay desde luego espacios turísticos en los que tanto turistas como anfitriones coinciden; no obstante, la forma en que son comprendidos y recorridos, así como los significados que les son atribuidos por cada uno, los hacen mucho más disímiles de lo que a simple vista pudiera pensarse. Esto es, existen asimetrías en el modo de caracterizar el territorio turístico. Por ello, el beneficio que puede o 
no recibir una comunidad por la presencia del turismo resulta muchas veces poco tangible.

El turismo se construye mediante la interacción entre turista y anfitrión, por lo tanto, el turismo se efectúa para el turista durante su tiempo de descanso y, para el anfitrión, en su tiempo de trabajo. Esta distinción de los tiempos tiene su origen en el concepto occidental de que el tiempo de trabajo es el que estructura la vida cotidiana de los pueblos y sus individuos. En el tiempo de descanso, relativamente marginal aunque creciente en los últimos dos siglos, surge el rol de turista. Producto de la interacción de ambos papeles, el turismo aparece como el encuentro de tiempos de descanso-trabajo. Desde un punto de vista analítico, el tiempo del turismo como construcción social se puede esquematizar en tres momentos: el de prefiguración, el de la interacción cara a cara y el que aquí se denominará de estructuración.

El momento de la prefiguración está orientado hacia la internalización de la realidad, hacia lo subjetivo. Tiene que ver con los períodos en los cuales no hay presencia física de la entidad alterna, esto es, cuando el turista no se encuentra frente al anfitrión y viceversa.

Cuando el turista no está ante el anfitrión, éste construye su realidad cotidiana con la perspectiva de que en el futuro se encontrará con aquél. Las actividades que realiza el anfitrión, que le confieren una identidad como tal, provienen de un acervo social de conocimiento que es compartido por otros anfitriones, y cada individuo las asume desde su historia personal, pero también desde la historia de su comunidad de vida. En el trabajo, son los compañeros, los colegas, los próximos significativos quienes muestran e indican las formas "correctas" de atender a los turistas, los aspectos que "son buenos" para ellos y lo que debe prepararse para atraerlos, atenderlos y obtener algo de su parte. Es claro que este momento se da en las comunidades anfitrionas de manera cotidiana, pues es parte de la vida de quienes dedican su actividad a la atención, organización y evaluación del turismo en los sitios de destino. Es un momento que, no obstante se origina en una dimensión social, sólo se establece y se formula en la subjetividad individual.

En el caso del turista, también ocurre este momento de prefiguración del turismo, que no exige la presencia física del anfitrión, incluso ni siquiera es necesario viajar. El solo hecho de que el individuo tenga la previsión de llevarlo a cabo y, por tanto, de establecer interacciones con anfitriones, es lo que distingue este momento. Las interacciones previstas son más o menos 
estereotipadas, con base en el acervo social de conocimiento con que se cuenta respecto a determinado destino turístico. Por ejemplo, el futuro turista prevé de manera distinta su papel como tal cuando viajará a un destino de playa que cuando irá a las montañas; qué actividad pretende realizar y la satisfacción que busca en cada caso serán completamente diferentes. Lo común en todo ello es la naturaleza de la predicción, que se hace respecto a lo que otros significativos, los turistas, han compartido directa o indirectamente; en este sentido, se tiene un referente social e histórico tanto en el nivel individual como en el comunitario, empero, sólo se formula en la subjetividad del turista. Es un momento orientado hacia el futuro, pues anticipa, prevé y adelanta, en ese instante es cuando el futuro turista comienza a construirse como tal, aunque lo haga en un sentido negativo, es decir, planteándose a sí mismo como no turista.

El segundo momento es aquel que, en la práctica, da origen al turismo; de hecho, es el punto de inicio de la realidad socialmente construida: la interacción cara a cara entre individuos. En el turismo, es la ocasión en que los roles dejan de ser subjetivos y son asumidos en la acción individual. Es el momento culminante del turismo, pues, aunque fugaz, es cuando se presenta con carácter objetivo en el mundo de la vida de cada individuo participante y su evidencia se da en el presente. Desde luego, las interacciones son múltiples y cada una persigue un fin distinto, por lo que cada individuo acude a un cúmulo de tipificaciones que se hacen evidentes en el uso de símbolos, lenguaje, actitudes y acciones que son tomados desde el acervo con que cuenta. Así pues, es un momento preestructurado, pero, a su vez, estructurante, pues con cada interacción los individuos aportan elementos novedosos, que no necesariamente provienen ni del acervo social, ni de la historia individual, quizá ni siquiera del momento de prefiguración del turismo, sino que tienen que ver con el nivel de la conciencia individual y con el contexto en el que sucede la interacción. Este momento singular, que implica la interacción de ambos roles, anfitrión y turista, es cuando en realidad existe de manera tangible el turismo en un sentido fenomenológico; es el tiempo en que el turismo se hace objetivo y es una realidad tangible y evidente, de allí su importancia central en esta propuesta. Es durante ese fugaz instante cuando el turista es turista y el anfitrión es anfitrión en toda la acepción con la que se identifica socialmente a estos roles.

El tercer momento del turismo es el de la estructuración, que tiende a hacerse permanente; en él, tanto turistas como anfitriones dejan de tener un significado individual, personal, y se transforman en actores típicos, que con sus 
actividades construyen entidades sociales, que se vuelven relativamente fijas a través del tiempo y de las constantes interacciones. Las manifestaciones físicas de este momento son las más evidentes, pues pueden ubicarse con claridad en el territorio, en los sitios de destino turístico. Son las que en otro campo se han llamado huella ecológica de la actividad humana. Por las manifestaciones físicas y por la estructuración de significados e identidades sociales, este momento tiende hacia la objetivación, hacia lo externo del sujeto. Éste es el lapso que queda registrado en la historia, en la identidad de la colectividad, en el carácter de los pueblos, tanto de quienes viajan (Hiernaux, 2000) como de quienes reciben a los viajeros. Es un tiempo que se acumula hacia el pasado.

El turismo surge de las interacciones tipificadas entre individuos que prefiguran, desempeñan, prevén o suponen los roles de turista y anfitrión. Desde esta perspectiva, el turismo es una construcción social -cuyo carácter histórico está ligado a la forma en que la sociedad da sentido a ambos papeles y a la interacción entre ellos-, que adquiere significados distintos en función del contexto histórico temporal y el espacio territorial en el que se ubique. Puesto que desde este punto de vista el turismo depende de la construcción social de los roles de turista y anfitrión es, entonces, un producto de la sociedad, aunque debería decirse con mayor propiedad que los "turismos" dependen de las diversas construcciones sociales de los roles de turista y anfitrión en comunidades y se reconstruyen, se recrean y se reproducen con cada momento de interacción.

La interacción turística tiene la capacidad de "objetivarse" mediante productos de la actividad humana, que se establecen más allá del lapso de interacción, esto significa que, a través de productos, se manifiesta la interacción, aun cuando no suceda en ese período.Algunos de estos productos son la significación (el uso de signos visuales para el turismo o el lenguaje del turismo), las prácticas que realizan los anfitriones y los turistas fuera del momento de la interacción, y los acervos sociales de conocimientos de los cuales dispone cada uno de los participantes en la interacción turística.

Realidad social objetiva del turismo: institucionalización y legitimación

Con su repetición, la actividad humana tiende a la habituación, aunque sin perder su significado. En el turismo, los viajes para los turistas y la atención al turista para el anfitrión suceden de forma habitual, lo cual hace innecesario que en cada interacción se defina la situación por parte de cada uno de los 
participantes, y abre la posibilidad para la deliberación y la innovación. Una vez que los actores tipifican de manera recíproca la acción habituada aparecen las instituciones. En el turismo, cuando anfitriones y turistas reconocen que determinada actividad es típicamente realizada por cierta clase de actor surgen instituciones como las de servicio al turista, las de regulación del turismo, las de promoción, etcétera.

Toda institución tiene una historia, no surge de inmediato en la interacción, y tiene que ver con el control de lo que determinada persona "debe" hacer en cierto momento. En esto reside lo tipificado de las acciones y de los actores: de forma recíproca las personas tienen acceso al conocimiento de lo que deben hacer frente al otro en una circunstancia específica, lo que da pie al orden social en el turismo.

Para la persona, las instituciones aparecen como algo "objetivo" que estaba allí antes que ella y que, muy probablemente, seguirá estando después de ella. En el turismo, las instituciones les son presentadas a los anfitriones y a los turistas de manera externa y coercitiva, de hecho sólo cuando aparece una segunda generación, esto es, cuando ni turistas ni anfitriones encuentran a la institución como algo permanente $y$ anterior a ellos.

Sin embargo, no toda tipificación conduce a que surjan instituciones, sólo aquellas que sedimentan de modo intersubjetivo, esto es, que además de tener un significado compartido por un grupo de individuos y de ser objetivada en un sistema de signos tienen la característica de ser compartidas únicamente de forma sistemática en un proceso de "educación" controlado por expertos, conocedores exclusivos del significado de las acciones. En el turismo, esto ocurre en las instituciones que cuentan con expertos, quienes se encargan de controlar y compartir los conocimientos institucionalizados a individuos seleccionados.

El turismo de la actualidad es un fenómeno social que se reconoce con el desempeño de dos roles fundamentales, el de anfitrión y el de turista. Ambos son construcciones sociales, en el sentido de que incluyen actividades, actitudes, prácticas, significados, representaciones, códigos y pautas de conducta que los identifican, $y$ son idealmente compartidos por muy diversos individuos a lo largo de distintas comunidades. En este tenor, los roles "existen" independientemente del individuo que los desempeñe, se han tipificado. Los roles constituyen tipificaciones de lo que son los individuos, y una distinción de aquello que no son. Una persona que cumple el papel de turista no es a la 
vez su anfitrión, del mismo modo que alguien que funge como anfitrión no es al mismo tiempo turista, aunque en ambos casos la ejecución del rol depende de la existencia del alterno y de que algún individuo lo desempeñe en una situación de interacción concreta.

El papel de anfitrión lo desempeñan diferentes actores sociales (González Damián, 2004); en primera instancia, quienes desempeñan el rol de manera activa, con el conocimiento de que su función se desarrolla en la interacción turística: los empresarios del turismo y de actividades económicas vinculadas a él, como los hoteleros, los restauranteros, los agentes de viajes, los operadores de servicios y atracciones turísticas, parques temáticos y museos; los empleados de toda la industria turística y de sectores ligados directa o indirectamente a ella; las dependencias e instancias de gobierno que fomentan y regulan la actividad; las instituciones educativas, los organismos de investigación, las organizaciones ciudadanas y los organismos internacionales que se ocupan de su análisis y fomento. Todos ellos constituyen su fragmento de realidad a partir de su rol como anfitrión, es decir, son anfitriones activos en la relación turística.

Por su parte, están también los anfitriones pasivos, quienes propiamente no llevan a cabo una función que los identifique como tales, sin embargo son quienes se constituyen como el espejo, el otro frente al que se identifica el turista. Se trata de los habitantes del destino turístico, a quienes en última instancia visitan los turistas y quienes de manera consciente o tácita permiten la visita de estos últimos y comparten sus recursos con ellos. Podría argumentarse, sin embargo, que estas personas, dado que no se constituyen a sí mismos como anfitriones, ya que no interactúan directa o conscientemente con los turistas, no tendrían que recibir esta denominación. No obstante, se configuran como el otro frente al que el turista se hace distinto,y de hecho, en la práctica, los residentes de una comunidad se identifican de manera automática como diferentes a los turistas o "fuereños" y, por tanto, distintos de ellos. En consecuencia, la relevancia de este grupo de personas es fundamental en la construcción del rol de turista y se ha optado por llamarlos anfitriones pasivos.

En el rol de turista se incorporan todos los viajeros que abandonan de forma temporal su lugar de residencia habitual y se trasladan a otro, en el que desempeñan esta función. Ser turista podría parecer un modo menor de ser, esto es, un rol social con el que apenas es necesario identificarse; empero, Hiernaux $(2000,99)$ hace un análisis detallado de lo que el tiempo dedicado al turismo en la vida cotidiana aporta a la construcción social de la realidad, y observa 
que "en una creciente valorización del tiempo del ocio, las sociedades actuales han aprendido que se puede enfrentar la lógica del trabajo, y generar espacios de mayor autonomía", con lo que destaca que la relevancia del turismo y del rol de turista van mucho más allá de la simple "puesta en escena" de un papel con el que nadie se reconoce. De hecho, si se preguntara a los viajeros fuera de su lugar de residencia si se identifican a sí mismos como turistas, pocos lo afirmarían, puesto que suponen que ser "turista" hace referencia a individuos que aceptan una oferta estandarizada y poco auténtica de servicios destinada a usuarios "poco exigentes". Sin embargo, ésta es una generalización hacia cierto tipo de turista.

Las instituciones interesadas en promover y mantener el turismo moderno son las que operan en el ámbito de los sistemas sociales conformados a partir de la racionalidad instrumental, que buscan el beneficio económico y que, por ende, se han desarrollado de una manera asimétrica, puesto que la acumulación de conocimiento tiende a concentrarse en pocas personas y grupos. En la búsqueda de mantenerse, las instituciones establecen procedimientos para permitirlo. Es aquí donde aparece con claridad la legitimación como proceso de objetivación de significado "de segundo orden", esto es, produciendo nuevos significados que sirven para integrar los ya atribuidos a procesos institucionales dispares.

Las instituciones que permanecen en el tiempo logran establecer una mezcla adecuada entre procesos de legitimación eficaces y la congruencia en términos de sentido con las actividades para las que fueron creadas. En el caso del turismo, existen instituciones que se mantienen y se han fortalecido, entre ellas las empresas de servicios de alojamiento, de restauración, las dependencias de promoción turística en el gobierno, los organismos internacionales como la Organización Mundial del Turismo, por mencionar algunas. Hay otras, aún con mayor fortaleza, que se entrelazan con campos simbólicos como el de las tradiciones, que aunque no se conforman como organismos sí establecen normas de comportamiento integradas a la cultura, por ejemplo la "hospitalidad". Las instituciones de este tipo son fuente de sentido y funcionan como estructuras sociales que permanecen a través de generaciones y entre diversas sociedades.

La realidad social subjetiva del turismo: internalización y socialización

En la medida en que la realidad social se ha internalizado, la persona tiende a "alejarse" de la interacción misma y ésta tiende a hacerse "menos real", por 
ello, para mantener viva la sensación de "realidad" en la subjetividad humana, es necesario que las interacciones sean repetitivas. Pero, en términos de sociedades es mediante procesos intersubjetivos que se preserva la realidad social subjetiva, por lo cual el diálogo entre individuos es el instrumento por excelencia. El diálogo y la existencia de estructuras de plausibilidad específicas para su mantenimiento son las herramientas de las que dispone la persona para conservar "viva" la realidad subjetiva. En el turismo, en la interacción se presenta el diálogo cara a cara entre turistas y anfitriones, y los contextos en los que se efectúa, los espacios turísticos y la temporalidad del turismo aportan las estructuras de plausibilidad necesarias; sin embargo, esto se está modificando en el mundo moderno.

La socialización deficiente no sólo sucede cuando hay una formación parcial mediada por otros, sino que puede ser resultado de las condiciones de la propia estructura social. Tal es el caso de la sociedad moderna, que ofrece a las personas múltiples esquemas de valores desde diversas instituciones, que por cierto cuentan con un sentido objetivo que se ubica en la esfera de la racionalidad instrumental pero que a la vez pretenden conectarse a valores de mayor orden, dirigidos a modificar u orientar el mundo de vida de las personas. Es el caso del conflicto, al que Jürgen Habermas denomina "colonización del mundo de la vida" y que Berger y Luckmann llaman "pluralismo moderno". En nuestro ámbito de estudio, esto sucede cuando las instituciones de turismo y las empresas pretenden modificar los valores y la cultura locales para que los empleados se adapten a un "estilo empresarial", pero lo que en realidad están haciendo es intentar legitimarse conectándose a valores del mundo de la vida cotidiana de la gente cuando su campo de sentido es puramente instrumental.

Es cierto que las personas, como individuos reflexivos, tienen la capacidad de crear y transformar en la interacción a las estructuras sociales, pero también es verdad que éstas existen y que de alguna manera condicionan las posibilidades individuales para formar parte de una comunidad de vida, de sentido $y$, en general, de una sociedad. Las instituciones, a pesar de su capacidad para autosostenerse, existen gracias a la competencia humana, así que el individuo puede modificarlas en favor de la sociedad y de las personas; los medios están a la vista y al alcance de todos, mediante la búsqueda de una mayor 
simetría entre las realidades subjetiva y objetiva, una mejor distribución del acervo social de conocimiento y la separación de áreas de acción institucional para evitar la superposición de esquemas de valores y de sentido. El turismo, como campo en el cual se favorece la libertad individual, tiene grandes posibilidades para actuar deliberadamente, para interactuar.

\section{Comentario final}

Durante casi dos siglos, el turismo se ha caracterizado como una actividad que las personas pueden llevar a cabo en su tiempo libre, esto es, cuando no tienen que cumplir con obligaciones de trabajo o relacionadas con él. El turismo se ha realizado en el tiempo de descanso, pues requiere una separación de la actividad cotidiana para llegar a un sitio de destino turístico. Hoy, sin embargo, las cosas pueden ser muy distintas; a través de las tecnologías de información y comunicación, los sitios distintos al propio son accesibles de forma inmediata y automática, lo cual tiene una doble implicación: por una parte, el turista ya no está obligado a desplazarse para acudir a su destino y disfrutar de sus paisajes, y, por la otra, cuando se dirige a un sitio turístico ya no necesita regresar a su lugar de trabajo para continuar con él. De hecho, muchos de los empleos actuales que sólo requieren una atención telefónica o virtual ya no demandan un espacio formal para ser desempeñados, por lo que el turista podría serlo de modo prácticamente permanente. Esto transforma de manera radical la concepción del turismo entendido como realizar viajes durante el tiempo libre. En realidad, el cambio es más profun-do aún, pues la línea que separa el tiempo libre del tiempo de trabajo se vuelve cada vez más tenue, cuando no desaparece por completo.

En consecuencia, vale la pena aproximarse al estudio del turismo desde un ángulo de sociología constructivista, puesto que los modelos estructurales pocas veces logran acercarse a lo que no es estructural, a la acción, a la dinámica permanente de la sociedad. Hoy día el turismo está dando muestras de sus transformaciones, de alteraciones que difícilmente pueden identificarse en un análisis sistémico, que antes bien busca las manifestaciones regulares, fijas y repetibles. 


\section{FUENTES CONSULTADAS}

Apostolopoulos, Y., S. Lerivaldi y A. Yianakis (1996). The Sociology of Tourism. Londres: Routledge.

Baert, Patrick (200l). La teoría social en el siglo xx. Trad. Jesús Cuéllar. Madrid: Alianza Editorial (Ciencias Sociales).

Berger, Peter L.y Thomas Luckmann (1968). La construcción social de la realidad. Trad. Silvia Zuleta. Buenos Aires:Amorrortu.

(1997) Modernidad, pluralismo y crisis de sentido. La orientación del hombre moderno. Barcelona: Paidós Studio.

Chadwick, Clifton B. (1993). Principios básicos de currículo: definición, constantes, enfoques y concepciones. Santiago:The Chadwick Group.

(2005) “La psicología de aprendizaje del enfoque constructivista”. Capítulo Internacional Red Global para la Mejora de la Performance. Sociedad Internacional para la Mejora de la Performance [en línea]. Disponible en: http://pignc-ispi.com/articles/education/chadwick-psicologia.htm

Cohen, Erik (1979). “A phenomenology of tourist experiences”. Sociology, I 3 (2), I79-20I.

(1984). “The Sociology of Tourism:Approaches, issues and findings". Annual Review of Sociology, vol. I0, enero-marzo, 373-392.

Corcuff, Philippe (1995). Las nuevas sociologías. Trad. Belén Urrutia. Madrid: Alianza Editorial (Ciencias Sociales).

Farrell, Bryan y Louise Twining-Ward (2004). "Reconceptualizing Tourism”. Annals of Tourism Research, 3I (2), 274-295.

Giddens, Anthony, et al. (1989). La teoría social hoy. México: Consejo Nacional para la Cultura y las Artes/Fondo de Cultura Económica.

González Damián,Alfonso (1999). "Turismo y vida cotidiana. El turismo desde el punto de vista de los actores sociales”. Convergencia, 6 (19), mayoagosto, 325-330 [Facultad de Ciencias Políticas y Administración Pública, Universidad Autónoma del Estado de México].

(2004). "Tiempos y espacios en el turismo del siglo XXI... hacia el ciberturismo". II Congreso Online del Observatorio para la Cibersociedad. Grupo 9: Cibergeografía/Geografía [en línea]. Disponible en: http://www.cibersociedad.net/congres2004/grups/fitxacom_publica2. php? grup $=9 \&$ \&id $=42$ I \&idioma $=$ es 
(2005). "El anfitrión como actor social en el turismo. Reflexiones desde el caso de Ixtapan de la Sal, México". Revista de Ciencias Sociales, III (I05), I55-I68 [Universidad de Costa Rica].

Habermas, Jürgen (1987). Teoría de la acción comunicativa, t. Il, Crítica de la razón funcionalista. Madrid:Taurus.

Heller, Agnes (1977). Sociología de la vida cotidiana, Barcelona: Península.

Hiernaux, Daniel (2000). "La fuerza de lo efímero.Apuntes sobre la construcción de la vida cotidiana en el turismo”, en Alicia Lindón (coord.). La vida cotidiana y su espacio-temporalidad. Barcelona:Anthropos/Centro Regional de Investigaciones Multidisciplinarias (CRIM)-Universidad Nacional Autónoma de México (UNAM)/El Colegio Mexiquense, 95-I 22.

Knebel, Hans Joachim (1984). Sociología del turismo. Cambios estructurales en el turismo moderno.Trad.José Ma. Núñez Espallargas. México y Barcelona: Editia Mexicana / Editorial Hispanoeuropea.

Leiper, N. (1990). Tourism Systems, Department of Management Systems, Ocassional Paper 2, Auckland: Massey University.

Lindón, Alicia (coord.) (2000). La vida cotidiana y su espacio-temporalidad. Barcelona:Anthropos / CRIM-UNAM/El Colegio Mexiquense.

Mannheim, K. (1952). Essays on Sociology of Knowledge. Londres: Routledge and Kegan Paul.

Matthews, M.R.( 1992)."OldWine in New Bottles:A Problem with Constructivist Epistemology”, en H. Alexander (ed.), Philosophy of Education 1992. Proceedings of the Forty-Eighth Annual Meeting of the Philosophy of Education Society. Urbana: Philosophy of Education Society, 303-3 I I. (1994) "Vino viejo en botellas nuevas: un problema con la epistemología constructivista". Enseñanza de las Ciencias, 12 (I), 79-88.

Mazón,Tomás (200I). Sociología del turismo. Madrid: Centro de Estudios Ramón Areces.

Osorio G., Maribel (2000). “Nuevos caminos para el estudio del turismo desde la teoría de sistemas”. Convergencia, 7 (23), septiembre-diciembre, 219-235.

(2004) “La complejidad del turismo”, ponencia presentada en el VI Congreso Nacional de Investigación Turística del Centro de Estudios Turísticos de la Secretaría de Turismo de México, Ciudad de México, octubre de 2004. 
Schütz,Alfred (1995). El problema de la realidad social. Comp. Maurice Natanson, trad. Néstor Miguez, $2^{\mathrm{a}}$ ed. Buenos Aires:Amorrortu. y Thomas Luckmann (1973). Las estructuras del mundo de la vida.Trad. Néstor Miguez. Buenos Aires:Amorrortu.

Searle, John (1997). La construcción de la realidad social. Barcelona: Paidós.

Smith, Valene S. (comp.) (1992). Anfitriones e invitados: antropología del turismo. Madrid: Endymion.

Tribe, J. (1997). “The indiscipline of Tourism”. Annals of Tourism Research 24 (3), 638-657.

Urry, J.(1991). “The sociology of Tourism”, en C.P. Cooper (ed.). Progress in Tourism, Recreation and Hospitality Management, vol. 3. Chichester: John Wiley and Sons, 48-57. 